\title{
Therapeutic targeting of cancer cell metabolism
}

\author{
Chi V. Dang, Max Hamaker, Peng Sun, Anne Le, and Ping Gao \\ Division of Hematology, Department of Medicine, Johns Hopkins University School of Medicine, \\ Ross 1032, 720 Rutland Avenue, Baltimore, MD 21212, USA
}

Chi V. Dang: cvdang@jhmi.edu

\begin{abstract}
In 1927, Otto Warburg and coworkers reported the increased uptake of glucose and production of lactate by tumors in vivo as compared with normal tissues. This phenomenon, now known as the Warburg effect, was recapitulated in vitro with cancer tissue slices exhibiting excessive lactate production even with adequate oxygen. Warburg's in vivo studies of tumors further suggest that the dependency of tumors in vivo on glucose could be exploited for therapy, because reduction of arterial glucose by half resulted in a four-fold reduction in tumor fermentation. Recent work in cancer metabolism indicates that the Warburg effect or aerobic glycolysis contributes to redox balance and lipid synthesis, but glycolysis is insufficient to sustain a growing and dividing cancer cell. In this regard, glutamine, which contributes its carbons to the tricarboxylic acid (TCA) cycle, has been re-discovered as an essential bioenergetic and anabolic substrate for many cancer cell types. Could alterations in cancer metabolism be exploited for therapy? Here, we address this question by reviewing current concepts of normal metabolism and altered metabolism in cancer cells with specific emphasis on molecular targets involved directly in glycolysis or glutamine metabolism.
\end{abstract}

\section{Keywords}

Warburg effect; Glycolysis; Glutaminolysis; Cancer therapy

\section{Metabolic profiles of normal cells and tissues}

The Warburg effect found in many cancer cells [1] should be considered in the context of the diverse metabolic profiles of normal human cells. The basal metabolic profiles of the normal cells are likely to influence the ultimate reprogramming of metabolism by altered oncogenes and tumor suppressors in cancer cells. Normal human cells vary dramatically according to their residence in tissues and organs, which themselves serve distinct functions for the body. Food substances absorbed through the gastrointestinal track are carried via the portal vein to the liver, where nutrients are stored or processed for circulation to other tissues. In this regard, the liver is organized into lobules, which contain a central vein and are surrounded by the portal triads. Hepatic arteries in the portal triad feed the lobules with oxygenated blood, whereas the portal vein in the triad carries nutrients from the intestine for processing such as the repackaging of fats as circulating lipoprotein particles or the storage of glucose as glycogen. When serum glucose level is low, glucagon released by the pancreas stimulates the conversion of hepatic glycogen to glucose, which is supplied via circulation to

(C) Springer-Verlag 2011

Correspondence to: Chi V. Dang, cvdang @ jhmi . edu.

Conflicts of interest

The authors declare no conflict of interests. 
other organs. Glucose is hence a key energetic currency for humans. After feeding, however, insulin is released from the pancreatic $\beta$ cells, when serum glucose level is high. Insulin, through its binding to cell surface receptors, triggers the import of glucose via transporters into tissues, such as muscle for utilization or liver for storage as glycogen. The heart, whose key function is to perfuse the body with oxygenated blood and nutrients, uses fatty acid oxidation as a key source of energy. The heart can also import lactate and convert it to pyruvate for oxidation in the mitochondrion and is hence considered as an oxidative organ. In contrast, skeletal muscle, whose major function is to provide mobility, uses glucose as a rapid energy source for oxidation in the mitochondrion. However, with prolonged exertion and limited oxygen supply, muscle can utilize anaerobic glycolysis to generate energy by converting glucose to lactate in the absence of oxygen (Fig. 1). Skeletal muscle is hence considered as an anaerobic organ. Through the Cori cycle, circulating lactate can be converted to pyruvate in the liver for gluconeogenesis.

Intriguingly, the brain, whose function is to process information, is the most metabolically active organ that is highly dependent on glucose [2]. Astrocytes import glucose, which could be stored as glycogen or catabolized by aerobic glycolysis to lactate (Fig. 1) [2]. Lactate is exported from astrocytes and then imported by neurons, converted to pyruvate that is oxidized by the mitochondria for ATP production. Hence, a commensal metabolic relationship between astrocytes and neurons is essential for normal brain function. The partnership between astrocytes and neurons extends to glutamine metabolism, whereby glutamine taken up by neurons is converted to glutamate as a neurotransmitter. Once released into the synaptic cleft, glutamate is imported into astrocytes and then recycled to glutamine by glutamine synthetase. Excessive glutamate is toxic to neurons, and hence, this symbiotic homeostasis between astrocytes and neurons is critically important for normal brain function. Disturbance in this circuitry has been implicated in the cause of neurodegenerative and neuropsychiatric disorders.

The liver detoxifies blood partly by removing ammonia, through the urea cycle and the synthesis of glutamine from glutamate and ammonia by glutamine synthetase that is enriched in the perivenous region of the liver lobule [3]. The muscle, which can be a major source for circulating glutamine with starvation, is also capable of glutamine synthesis when nutrients are scarce. The kidney, on the other hand, uses glutamine to produce ammonia for the regulation of blood $\mathrm{pH}$. The body also uses fatty acids as a fuel source via fatty acid oxidation. Acetyl-CoA derived from fatty acids requires carbohydrate or glutamine catabolites to enter into the tricarboxylic acid (TCA) cycle to form citrate, which permits the oxidation of acetyl-CoA (Fig. 1). Under starved conditions when glucose is depleted, oxaloacetate is consumed to form glucose through gluconeogenesis, and is hence unavailable for fatty acid oxidation. Under these conditions, acetyl-CoA is converted to acetoacetate and 3-hydroxybutyrate (known as ketone bodies), which are normally used by the heart and kidney cortex under non-starved conditions. The brain, however, adapts and uses ketone bodies under prolonged starvation conditions. Thus, normal cells have remarkably varied metabolic profiles, which could be reprogrammed with starvation. The conversion of normal cells to cancer cells is accompanied by the metabolic profile of the cell of origin, unless the cancer cells have almost fully de-differentiated and adopt the Warburg effect or aerobic glycolysis that characterizes metabolism in early embryogenesis.

Rapid cell division, early in embryogenesis, is characterized by aerobic glycolysis with the utilization of glucose and production of lactate until the 16-cell stage when the embryonic genome is activated [4]. At this juncture with a rapid increase in embryonic growth, both aerobic glycolysis and oxidative phosphorylation occur concurrently. However, whether different layers of the embryo use different modes of metabolism is not known. Glucose, pyruvate, lactate and glutamine are all used by the growing embryo (Fig. 1). As such, the 
undifferentiated state of early embryos is associated with increased aerobic glycolysis, which is then accompanied by oxidative phosphorylation, and the use of glutamine as a nitrogen source when rapid growth is accompanied by the activation of the embryonic genome.

\section{Current concepts of the Warburg effect}

The Warburg effect, which describes the propensity of cancer cells to use glucose at a high level and convert it to lactate, dominated our conceptual framework of altered cancer cell metabolism over many decades (Fig. 1) [1]. However, the Warburg effect and altered cancer cell metabolism were subjects of sharp criticism over the past five decades and were further neglected when modern molecular oncology emerged on the scene of cancer research. In fact, the promise of a richer, deeper understanding of the molecular causes of cancer came with the discoveries of oncogenes and tumor suppressor genes [5]. The genetic basis of cancer provided by these discoveries has provided profound insights into the molecular circuitries that control the growth and proliferation of normal cells. When these pathways are short-circuited or become deranged with oncogene activation and loss of tumor suppressors, cancer cells proliferate without regard for external cellular cues that would normally attenuate the proliferation of normal cells. The paradigm generated by altered cancer genes consumed the community of cancer researchers over three decades, a period when cell autonomous genetic alterations were believed to be the key driving force for cancer development and progression. Indeed, changes in cellular metabolism associated with oncogene activation or loss of tumor suppressors were believed to be epiphenomena of the more important genetic alterations, which drive the cell cycle.

Alterations of oncogenes and tumor suppressors per se, cause cell growth and division to be deregulated such that cell-cell contact and other external cues no longer abrogate cell proliferation. The drive to acquire cell mass and proliferate, however, requires an energy supply and building blocks [6]. In fact, tumors could not progress in vivo beyond a diameter of about $1 \mathrm{~mm}$ without new blood vessels recruited into the tumor mass [7]. With the recognition that tumor-associated neo-angiogenesis is essential for tumor progression, the prevailing concept of genetic alterations being sufficient to trigger tumorigenesis was revised. In essence, a growing tumor requires raw building blocks and a source of energy to self-replicate. In fact, the enhanced tumor uptake of radiolabeled fluoro-2-deoxyglucose in positron emission tomography (PET) scans supports the increased uptake of glucose by tumors. Indeed, Warburg illustrated through in vivo and ex vivo studies that many human and animal cancer tissue sections had enhanced glucose uptake and lactate production [1]. It is critically important to note, however, that cells are not made of just carbon, hydrogen, and oxygen which are provided by glucose, but they also comprise nitrogen, phosphorus, sulfur, as well as many minerals. As such, the Warburg effect is insufficient to sustain the growth and proliferation of a cancer, which requires the import of other nutrients as building blocks for cell division.

Although angiogenesis supplies a growing tumor with oxygen and nutrients, the neovasculature is poorly formed often with leaky blood vessels that do not link the arterial to the venous circulation but is rather dead-end [8]. As such, the tumor tissue tends to be poorly perfused as compared to the normal tissue counterpart. The inadequate perfusion results in pervasive tumor tissue hypoxia that induces the stabilization of HIF-1a (Fig. 2), which is constitutively translated and degraded in an oxygen-dependent manner [9]. HIF-1a dimerizes with HIF-1 $\beta$ to form a transcription factor that activates many enzymes involved in glycolysis and suppresses genes involved in mitochondrial biogenesis. HIF-1 further induces angiogenesis and is often increased in cancers either as an adaptive measure or as the result of signal transduction pathways that stabilize HIF-1a independent of oxygen. 
Although activation of HIF-1 in cancers reprograms tumor metabolism toward glycolysis, hypoxic adaptation was not considered by Warburg, who carefully designed in vitro experiments such that oxygen was not limiting. The Warburg effect is defined as the enhanced used of glucose and its conversion to lactate by cancer cells even when oxygen is adequate, a process that is also known as aerobic glycolysis. This contrasts with the classical switch of glucose metabolism to anaerobic glycolysis when oxygen is inadequate. The contributions of hypoxic cellular adaptation and cell autonomous oncogenic alterations to cancer cell metabolism are both important for the understanding of cancer cell biology. These issues have been at the crossroads of debate over the years and cause for confusion.

What is the molecular basis for the Warburg effect, the ability of cancer cells to undergo aerobic glycolysis in a fashion that oxygen cannot inhibit glycolysis as it could with normal cells? Are the functions of oncogenes and tumor suppressors linked to aerobic glycolysis or the Warburg effect? In general, activation of oncogenes, such as Ras, Akt, and Myc or inactivation of $\mathrm{p} 53$, can contribute to the Warburg effect in a HIF-1-independent fashion $[10,11]$. However, many oncogenic pathways, including Ras, Akt, PI3K, and VHL, converge on HIF-1, causing the nonhypoxic expression of HIF-1, which confers a transcriptional program of glycolysis [12]. In this regard, the inability of oxygen to suppress glycolysis or the Warburg effect can be triggered by oncogenes independently of, or through HIF-1.

Cancer cells, similar to normal cells, live in niches and microenvironments that are heterogeneous. Specifically, gradients of oxygen and nutrients can be steep, being elevated in concentrations near new tumor blood vessels and diminished away from the blood [13] (Fig. 2). As such, in addition to the cell-autonomous genetic alterations that define the cancer cell, the altered genomic and metabolic networks are also perturbed by the availability of nutrients and oxygen. Indeed, it was demonstrated recently that tumor cells can extrude lactate, which could then be recycled to pyruvate for mitochondrial oxidation by tumor cells that use oxidative phosphorylation and are presumably nearby blood vessels [14]. This model suggests that even if the cell autonomous changes in cancer cells cause them to rely primarily on oxidative phosphorylation, the hypoxic microenvironment in areas away from the neo-vascular would induce glycolysis in the hypoxic tumor cells (Fig. 2) [15]. While stimulation of glycolysis is a common outcome of altered cancer genes, a number of experimental systems suggest that cancer cells can have a metabolic profile favoring oxidative phosphorylation. However, the in vivo hypoxic tumor microenvironment appears to play a profound role in modulating tumor cell metabolism. Specifically, how hypoxia influences mitochondrial homeostasis and metabolism in cancer cells will be important to understand as we seek strategies to target metabolism for cancer therapy.

The prevailing concept that mitochondrial function is necessarily decreased in cancers or with the Warburg effect needs revision [15]. Warburg's observation in 1927 indicated that cancers still consume oxygen and respire, but unlike normal tissues, the cancers extruded large amounts of lactate into the veins [1]. In fact, concurrent aerobic glycolysis and oxidative phosphorylation occur in experimental systems with activated oncogenes, with glutamine playing an important role in oxidative phosphorylation and anapleurosis. Because glucose and glutamine only provide the cell with the elements $\mathrm{C}, \mathrm{H}, \mathrm{O}$, and $\mathrm{N}$, other nutrients containing $\mathrm{S}$ and $\mathrm{P}$, as well as numerous ions, must also be imported for the synthesis of a new cell. A complete understanding of cancer cell metabolism beyond Warburg's contribution to cancer research will require further studies on other nutrients in addition to glucose and glutamine. Furthermore, cancer cell metabolism is influenced by the cell of origin imprinted with a metabolic profile reflective of its normal function. Hence, cell type specific effects and the degree of dedifferentiation of cancer cells are likely to affect the overall metabolic phenotype of a cancer cell with the expectation that highly 
undifferentiated cancer cells tend to have a heightened Warburg effect, which provides the basis for targeting metabolism in cancer therapy.

\section{Targeting the Warburg effect}

Given that cancer cells have heightened aerobic glycolysis and normal proliferating cells also display the Warburg effect, how tenable is it to target cancer metabolism? A distinct difference between cancer cells and normal cells resides in the oncogenic activation of deregulated biomass accumulation independent of nutrient availability to the cancer cells. As such, activation of oncogenes or loss of tumor suppressors renders cancer cells addicted to an adequate supply of bioenergetic and anabolic substrates for continued growth and proliferation $[16,17]$. When the bioenergetic demand for biomass accumulation outstrips bioenergetic supply, cancer cells undergo autophagy and, ultimately, a bioenergetic cell death. In this regard, key metabolic regulators and enzymes that are conduits for bioenergetic supply for the cancer could be therapeutic targets with sufficient therapeutic windows that when targeted would spare normal tissues [18].

Hexokinase 2 (HK2) (Fig. 1), which is now documented to be a transcriptional target of HIF-1 and Myc, has been deemed a therapeutic target for many years starting with its discovery as a mitochondrially associated enzyme. Once glucose is transported into the cell, it is phosphorylated by HK2 and is retained intracellularly by the acquisition of negative charges. Glucose-6-phosphate is then converted to fructose-6-phosphate by glucose phosphate isomerase, which is also known as autocrine motility factor. Over the years, 3bromopyruvate was thought to target HK2, although recent proteomic analyses suggest that 3-bromopyruvate targets glyceraldehydes 3-phosphate dehydrogenase (GAPDH) [19]. Nonetheless, 3-bromopyruvate-despite its lack of specificity and strong alkylation potential—appears to have significant anti-cancer effect in vivo. More specific inhibitors to HK2 are not yet available to determine whether specific targeting of HK2 is feasible and tolerable, given that hexokinase provides the essential first step in glucose catabolism.

6-Phosphofructo-1-kinase (PFK), downstream of HK2 in glycolysis, converts fructose-6phosphate to fructose-1,6-bisphosphate. PFK is allosterically regulated with ATP being a potent inhibitor and fructose-2,6-bisphosphate (F2,6-BP), a potent activator. F2,6-BP levels are affected by four enzymes of the 6-phosphofructo-2-kinase/fructose-2,6-bisphosphatase (PFKFB 1-4) family, such that diminished F2,6-BP level could decrease PFK activity and favor the shunting of glucose-6-phosphate into the pentose phosphate pathway (PPP) and elevated F2,6-BP level enhances glycolysis. PFKFB3, a potent target of HIF-1, produces F2,6-BP and favors glycolysis. A small molecule inhibitor of PFKFB3 (3-(3-pyridinyl)-1-(4pyridinyl)-2-propen-1-one (3PO)) was documented to inhibit glycolysis in vitro and xenograft tumor growth in vivo [20]. The PFKFB family of enzymes is likely to play a critical role in cancer metabolism, and hence, would be of interest for targeted therapy.

Pyruvate kinase (PK) (Fig. 1), whose product converts phosphoenolpyruvate to pyruvate with release of an ATP, is a target gene of Myc and HIF-1 with two splice variant mRNAs. The splicing variant PKM2, which is favored by a Myc-induced splicing factor, has been linked to altered cancer metabolism for over a decade, but recent interest in this potential target was heightened by its rediscovery as a critical contributor to the Warburg effect. A major confusion still exists in that PKM1, the splice variant found in many normal cells, is more catalytically active than PKM2, which is elevated in cancers. Nonetheless, unlike PKM1, PKM2 is allosterically positively regulated by fructose-2,6-bisphosphate, which is displaced by tyrosine phosphorylated peptides in actively dividing cells [21]. Hence, PKM2 itself does not contribute to the Warburg effect per se, rather another pathway for the generation of pyruvate from phosphoenol pyruvate has been proposed to account for the 
increased aerobic glycolysis seen with PKM2 expression [22]. Given that activated PKM2 slows glycolysis, efforts are being made to explore that potential use of PKM2 inhibitors for therapeutic purposes [23]. It is currently too early to tell whether this approach will be fruitful.

Lactate dehydrogenase A (LDHA) (Fig. 1), which is a target gene of Myc and HIF-1, has been demonstrated to be essential for tumorigenesis by gene knockdown in three independent studies, after it was first documented to be essential for human Burkitt lymphoma clonogenicity [10, 24]. A small-molecule inhibitor of LDHA (FX11; 3dihydroxy-6-methyl-7-(phenylmethyl)-4-propylnaphthalene-1-carboxylic acid) was demonstrated to trigger oxidative stress in cancer cells similar to gene knockdown mediated by siRNA, resulting in necrotic cell death [9]. Reduction of LDHA activity was associated with an elevation of NADH/NAD+ ratio, which was linked with increased reactive oxygen species (ROS) and cell death. It is surmised that excess NADH could diminish upstream glycolytic flux, which requires recycled NAD+, and increase inappropriate respiratory complex I activity and ROS production, resulting in cell death that was partially rescued by the antioxidant $\mathrm{N}$-acetylcysteine. Because LDHA is essential for the production of lactate in the Warburg effect, its inhibition targets the heart of aerobic glycolysis.

The Warburg effect is enhanced by Myc and HIF-1 through their induction of pyruvate dehydrogenase kinase 1 (PDK1) (Fig. 1), which phosphorylates and inactivates pyruvate dehydrogenase (PDH). Inhibition of PDH diminishes the conversion of pyruvate to acetylCoA, allowing more pyruvate to be converted to lactate. PDK1 appears pivotal for the regulation of redox balance through titrating pyruvate flux into the mitochondrion as a function of oxygen availability. When oxygen is low, continued pyruvate flux to acetyl-CoA triggers increased ROS production and decreased cell survival. In this regard, inhibition of PDK by dichloroacetate (DCA) that has been used clinically to manage congenital lactic acidosis appears to have an anti-tumor effect preclinically in select tumor models. DCA was also documented anecdotally to affect metabolism of human glioblastomas. Controlled randomized clinical studies are needed to critically assess the effectiveness of DCA in human cancers [25].

It is notable that while the Warburg effect is mediated by known critical enzymes in glucose metabolism, regulated by HIF-1 and Myc, inhibition of at least two (LDHA and PDK1) result in redox stress and cell death. These observations suggest that while glucose metabolism itself is affected by inhibition of these enzymes, a profound effect, however, is on redox homeostasis. In this regard, it is notable that glucose-6-phosphate (produced by HK2) can be catabolized through the PPP to produce ribose and NADPH, a key catabolite for reductive metabolism (Fig. 1). As such, enzymes in the PPP, such as transketolase (TKT), have been proposed to be a therapeutic target, and thiamine analogs have been proposed as transketolase inhibitors [26]. In vivo proof-of-concept preclinical studies, however, failed to demonstrate a therapeutic effect with HCT 116 xenografts [26].

\section{Glutamine: the other Achilles' heel in cancer metabolism}

The dependency of cancer cells on glutamine was not fully appreciated by Warburg some 80 years ago. More recently, it was documented that Myc regulates many steps in glutamine metabolism, suggesting that key points in glutamine metabolism could be targeted when metabolism is reprogrammed in cancer cells. In this regard, it is notable that while Myc could post-transcriptionally increase glutaminase (GLS) (Fig. 1), which converts glutamine to glutamate that is in turn catabolized to a-ketoglutarate to feed the TCA cycle, activated RhoGTPase can also increase GLS for neoplastic transformation. In screening for inhibitors of RhoGTPase mediated transformation, a small molecule 968 was found to inhibit 
transformation through inhibition of GLS [27]. Compound 968, but not its inactive derivative, was also able to inhibit in vivo lymphoma-genesis by a Myc-inducible human Burkitt model. This finding underscores the theme that critical enzyme nodes for cancer metabolism are influenced by multiple oncogenic pathways.

Reprogramming of cancer cell metabolism by isocitrate dehydrogenase (IDH) (Fig. 1) mutations, frequently found in human gliomas and acute myelogenous leukemia, is also exploitable for therapy $[28,29]$. Specifically, IDH1 and IDH2 mutations result in a neoenzyme activity that converts a-ketoglutarate to 2-hydroxyglutarate rather than the normal reaction of IDH resulting in the conversion of isocitrate to a-ketoglutarate [30]. Thus, it is conceivable that inhibitors of mutant IDH might be therapeutically useful if cancer cells with mutant IDH are dependent on the mutant enzyme for their cancer phenotype. It is unclear at this stage whether mutant IDHs are tumor initiators, which likely perturb the epigenome since a-ketoglutarate is required for histone and DNA demethylases, and hence are not necessary for tumor maintenance. The reprogramming of metabolism by mutant IDH, particularly through increased production of 2-hydroxyglutarate from glutamine, suggest that cancer cells with mutant IDHs are addicted to glutamine as a source for aketoglutarate that is then converted to 2-hydroxyglutarate. The concept was tested by inhibition of glutaminase by siRNA-mediated knock-down and a small-molecule inhibitor (BPTES; bis-2-(5-phenyl-acetamido-1,2,4-thiadiazol-2-yl)ethyl sulfide) [31]. Both approaches caused a more significant delay in the growth of glioma cells engineered to inducibly express mutant IDH1 as compared to those expressing wild-type IDH1. This study suggests that reprogrammed metabolism in cancer cells can be targeted.

Glutamine metabolism involves the conversion of glutamate to a-ketoglutarate, mediated by two types of enzymes: glutamate dehydrogenase (GLUD1) and aminotransferases (glutamate pyruvate transaminase (GPT), or glutamate oxalo-acetate transaminase (GOT)). While GLUD1 deaminates glutamate and can be inhibited by the green tea component epigallocatechin-3-gallate (EGCG) [32], the transaminases are inhibited by aminooxyacetate (AOA). EGCG has been documented to have anti-tumor effects in vivo, but its precise target is unclear given that many candidate molecular targets have been reported [33]. Intriguingly, AOA, which has been used in humans, appears to have significant inhibitory in vivo effect on a breast cancer xenograft [34]. It is currently unclear, however, which of the two types of enzymes (GLUD1 versus transaminases) are used for glutamine metabolism in specific types of cancers. It is likely that the variability in the uses of these two pathways by cancers to produce a-ketoglutarate would contribute to differential sensitivities to EGCG or AOA.

\section{Concluding remarks}

Renewed interest in cancer metabolism has generated new hopes that a new class of therapeutic agents may finally appear on the stage for cancer therapy. However, it should be noted as alluded to in the beginning of this review that metabolic profiles of cancers are likely to be influenced by the cell of origin, which varies from tissue to tissue. The heterogeneity of metabolic profiles of even a single type of cancer, such as pancreatic cancer for example, must be better understood before targeting cancer could be realized for the more deadly and currently untreatable cancers. For other cancers with more uniform phenotypes, such as Burkitt lymphoma that does not lack an effective therapeutic regimen, it is envisioned that our richer understanding of refractory lymphomas and their metabolic profiles may lead to the effective use of metabolic inhibitors clinically. Not discussed in this review are molecules that affect regulators of metabolism, such as inhibitors or activators of HIF-1, mTOR, and AMP kinase. Specifically, mTOR inhibitors have provided tangible proof-of-concept that cancer metabolism is targetable in the clinic, albeit with variable outcomes. Finally, metformin, an anti-diabetic drug, has emerged as another clinically 
tangible metabolic inhibitor through epidemiological studies of cancers [35]. Metformin, through its inhibition of the mitochondrial complex I, induces AMPK, which is believed to be associated with a lower rate of cancer development [36]. There are now over a dozen clinical trials using metformin for cancer therapy. In the next 5 years, the renewed interest in cancer metabolism is likely to enhance our understanding as well as providing a new class of anti-cancer drugs.

\section{Acknowledgments}

Our work is partially supported by the Stand-Up-to-Cancer AACR Translational Research grant, National Cancer Institute, and the Leukemia \& Lymphoma Society.

\section{References}

1. Warburg O, Wind F, Negelein E. The metabolism of tumors in the body. J Gen Physiol. 1927; 8:519-530. [PubMed: 19872213]

2. Benarroch EE. Glycogen metabolism: metabolic coupling between astrocytes and neurons. Neurology. 2010; 74:919-923. [PubMed: 20231669]

3. Burke ZD, Reed KR, Phesse TJ, Sansom OJ, Clarke AR, Tosh D. Liver zonation occurs through a beta-catenin-dependent, c-Myc-independent mechanism. Gastroenterology. 2009; 136(23162324):e2311-e2313.

4. Khurana NK, Niemann H. Energy metabolism in preim-plantation bovine embryos derived in vitro or in vivo. Biol Reprod. 2000; 62:847-856. [PubMed: 10727252]

5. Fearon ER, Vogelstein B. A genetic model for colorectal tumorigenesis. Cell. 1990; 61:759-767. [PubMed: 2188735]

6. Deberardinis RJ, Sayed N, Ditsworth D, Thompson CB. Brick by brick: metabolism and tumor cell growth. Curr Opin Genet Dev. 2008; 18:54-61. [PubMed: 18387799]

7. Hanahan D, Weinberg RA. The hallmarks of cancer. Cell. 2000; 100:57-70. [PubMed: 10647931]

8. Fukumura D, Duda DG, Munn LL, Jain RK. Tumor microvasculature and microenvironment: novel insights through intravital imaging in pre-clinical models. Microcirculation. 2010; 17:206-225. [PubMed: 20374484]

9. Le A, Cooper CR, Gouw AM, Dinavahi R, Maitra A, Deck LM, Royer RE, Vander Jagt DL, Semenza GL, Dang CV. Inhibition of lactate dehydrogenase A induces oxidative stress and inhibits tumor progression. Proc Natl Acad Sci U S A. 2010; 107:2037-2042. [PubMed: 20133848]

10. Shim H, Dolde C, Lewis BC, Wu CS, Dang G, Jungmann RA, Dalla-Favera R, Dang CV. c-Myc transactivation of LDH-A: implications for tumor metabolism and growth. Proc Natl Acad Sci U S A. 1997; 94:6658-6663. [PubMed: 9192621]

11. Levine AJ, Puzio-Kuter AM. The control of the metabolic switch in cancers by oncogenes and tumor suppressor genes. Science. 2010; 330:1340-1344. [PubMed: 21127244]

12. Semenza GL. HIF-1: upstream and downstream of cancer metabolism. Curr Opin Genet Dev. 2010; 20:51-56. [PubMed: 19942427]

13. Dang CV. Rethinking the Warburg effect with Myc micromanaging glutamine metabolism. Cancer Res. 2010; 70:859-862. [PubMed: 20086171]

14. Sonveaux P, Vegran F, Schroeder T, Wergin MC, Verrax J, Rabbani ZN, De Saedeleer CJ, Kennedy KM, Diepart C, Jordan BF, Kelley MJ, Gallez B, Wahl ML, Feron O, Dewhirst MW. Targeting lactate-fueled respiration selectively kills hypoxic tumor cells in mice. J Clin Invest. 2008; 118:3930-3942. [PubMed: 19033663]

15. Fogal V, Richardson AD, Karmali PP, Scheffler IE, Smith JW, Ruoslahti E. Mitochondrial p32 protein is a critical regulator of tumor metabolism via maintenance of oxidative phosphorylation. Mol Cell Biol. 2010; 30:1303-1318. [PubMed: 20100866]

16. Shim H, Chun YS, Lewis BC, Dang CV. A unique glucose-dependent apoptotic pathway induced by c-Myc. Proc Natl Acad Sci U S A. 1998; 95:1511-1516. [PubMed: 9465046] 
17. Yuneva M, Zamboni N, Oefner P, Sachidanandam R, Lazebnik Y. Deficiency in glutamine but not glucose induces MYC-dependent apoptosis in human cells. J Cell Biol. 2007; 178:93-105. [PubMed: 17606868]

18. Tennant DA, Duran RV, Gottlieb E. Targeting metabolic transformation for cancer therapy. Nat Rev Cancer. 2010; 10:267-277. [PubMed: 20300106]

19. Ganapathy-Kanniappan S, Vali M, Kunjithapatham R, Buijs M, Syed LH, Rao PP, Ota S, Kwak BK, Loffroy R, Geschwind JF. 3-Bromopyruvate: a new targeted antiglycolytic agent and a promise for cancer therapy. Curr Pharm Biotechnol. 2010; 11:510-517. [PubMed: 20420565]

20. Clem B, Telang S, Clem A, Yalcin A, Meier J, Simmons A, Rasku MA, Arumugam S, Dean WL, Eaton J, Lane A, Trent JO, Chesney J. Small-molecule inhibition of 6-phosphofructo-2-kinase activity suppresses glycolytic flux and tumor growth. Mol Cancer Ther. 2008; 7:110-120. [PubMed: 18202014]

21. Christofk HR, Vander Heiden MG, Wu N, Asara JM, Cantley LC. Pyruvate kinase M2 is a phosphotyrosine-binding protein. Nature. 2008; 452:181-186. [PubMed: 18337815]

22. Vander Heiden MG, Locasale JW, Swanson KD, Sharfi H, Heffron GJ, Amador-Noguez D, Christofk HR, Wagner G, Rabinowitz JD, Asara JM, Cantley LC. Evidence for an alternative glycolytic pathway in rapidly proliferating cells. Science. 2010; 329:1492-1499. [PubMed: 20847263]

23. Vander Heiden MG, Christofk HR, Schuman E, Subtelny AO, Sharfi H, Harlow EE, Xian J, Cantley LC. Identification of small molecule inhibitors of pyruvate kinase M2. Biochem Pharmacol. 2010; 79:1118-1124. [PubMed: 20005212]

24. Qing G, Skuli N, Mayes PA, Pawel B, Martinez D, Maris JM, Simon MC. Combinatorial regulation of neuroblastoma tumor progression by $\mathrm{N}-\mathrm{Myc}$ and hypoxia inducible factor HIF-1alpha. Cancer Res. 2010; 70:10351-10361. [PubMed: 20961996]

25. Michelakis ED, Sutendra G, Dromparis P, Webster L, Haromy A, Niven E, Maguire C, Gammer TL, Mackey JR, Fulton D, Abdulkarim B, McMurtry MS, Petruk KC. Metabolic modulation of glioblastoma with dichloroacetate. Science Translational Medicine. 2010; 2:31ra34.

26. Thomas AA, Le Huerou Y, De Meese J, Gunawardana I, Kaplan T, Romoff TT, Gonzales SS, Condroski K, Boyd SA, Ballard J, Bernat B, DeWolf W, Han M, Lee P, Lemieux C, Pedersen R, Pheneger J, Poch G, Smith D, Sullivan F, Weiler S, Wright SK, Lin J, Brandhuber B, Vigers G. Synthesis, in vitro and in vivo activity of thiamine antagonist transketolase inhibitors. Bioorg Med Chem Lett. 2008; 18:2206-2210. [PubMed: 18267359]

27. Wang JB, Erickson JW, Fuji R, Ramachandran S, Gao P, Dinavahi R, Wilson KF, Ambrosio AL, Dias SM, Dang CV, Cerione RA. Targeting mitochondrial glutaminase activity inhibits oncogenic transformation. Cancer Cell. 2010; 18:207-219. [PubMed: 20832749]

28. Parsons DW, Jones S, Zhang X, Lin JC, Leary RJ, Angenendt P, Mankoo P, Carter H, Siu IM, Gallia GL, Olivi A, McLendon R, Rasheed BA, Keir S, Nikolskaya T, Nikolsky Y, Busam DA, Tekleab H, Diaz LA Jr, Hartigan J, Smith DR, Strausberg RL, Marie SK, Shinjo SM, Yan H, Riggins GJ, Bigner DD, Karchin R, Papadopoulos N, Parmigiani G, Vogelstein B, Velculescu VE, Kinzler KW. An integrated genomic analysis of human glioblastoma multiforme. Science. 2008; 321:1807-1812. [PubMed: 18772396]

29. Reitman ZJ, Yan H. Isocitrate dehydrogenase 1 and 2-mutations in cancer: alterations at a crossroads of cellular metabolism. J Natl Cancer Inst. 2010; 102:932-941. [PubMed: 20513808]

30. Dang L, White DW, Gross S, Bennett BD, Bittinger MA, Driggers EM, Fantin VR, Jang HG, Jin S, Keenan MC, Marks KM, Prins RM, Ward PS, Yen KE, Liau LM, Rabinowitz JD, Cantley LC, Thompson CB, Vander Heiden MG, Su SM. Cancer-associated IDH1 mutations produce 2hydroxyglutarate. Nature. 2009; 462:739-744. [PubMed: 19935646]

31. Seltzer MJ, Bennett BD, Joshi AD, Gao P, Thomas AG, Ferraris DV, Tsukamoto T, Rojas CJ, Slusher BS, Rabinowitz JD, Dang CV, Riggins GJ. Inhibition of glutaminase preferentially slows growth of glioma cells with mutant IDH1. Cancer Res. 2010

32. Li M, Smith CJ, Walker MT, Smith TJ. Novel inhibitors complexed with glutamate dehydrogenase: allosteric regulation by control of protein dynamics. J Biol Chem. 2009; 284:22988-23000. [PubMed: 19531491] 
33. Yang CS, Wang X, Lu G, Picinich SC. Cancer prevention by tea: animal studies, molecular mechanisms and human relevance. Nat Rev Cancer. 2009; 9:429-439. [PubMed: 19472429]

34. Thornburg JM, Nelson KK, Clem BF, Lane AN, Arumugam S, Simmons A, Eaton JW, Telang S, Chesney J. Targeting aspartate aminotransferase in breast cancer. Breast Cancer Res. 2008; 10:R84. [PubMed: 18922152]

35. Decensi A, Puntoni M, Goodwin P, Cazzaniga M, Gennari A, Bonanni B, Gandini S. Metformin and cancer risk in diabetic patients: a systematic review and meta-analysis. Cancer Prev Res (Phila). 2010; 3:1451-1461. [PubMed: 20947488]

36. Owen MR, Doran E, Halestrap AP. Evidence that metformin exerts its anti-diabetic effects through inhibition of complex 1 of the mitochondrial respiratory chain. Biochem J. 2000; 348(Pt 3):607614. [PubMed: 10839993] 


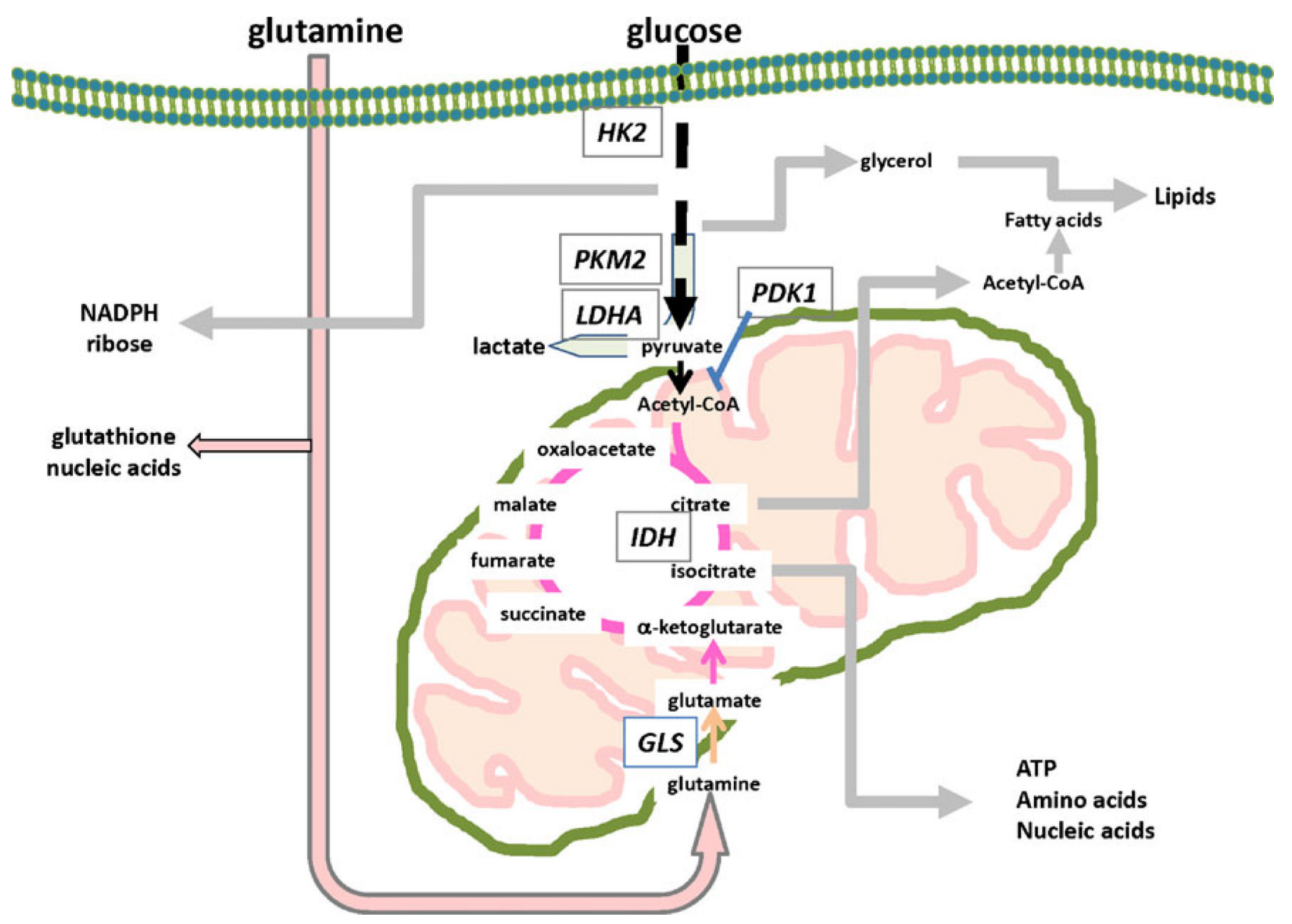

Figure 1.

Glucose and glutamine metabolic pathways involved in cancer cell metabolism. Glucose and glutamine are depicted to be transported into the cytoplasm for catabolism through glycolysis and the tricarboxylic acid cycle. Glucose is converted to trioses, which contributes to lipid synthesis, through the action of enzymes such as hexokinase 2 (HK2), pyruvate kinase M2 (PKM2), and lactate dehydrogenase A (LDHA). Pyruvate dehydrogenase kinase 1 (PDK1), which is activated by HIF-1, inhibits the conversion of pyruvate to acetyl-CoA. Glutamine is converted to glutamate by glutaminase (GLS) and then further catabolized to a-ketoglutarate to enter the TCA cycle, which includes isocitrate dehydrogenase (IDH). Anabolic carbons from the TCA cycle contribute to the synthesis of amino acids, lipids, and nucleic acids. Glutamine is also depicted to contribute to glutathione and nucleic acid synthesis 


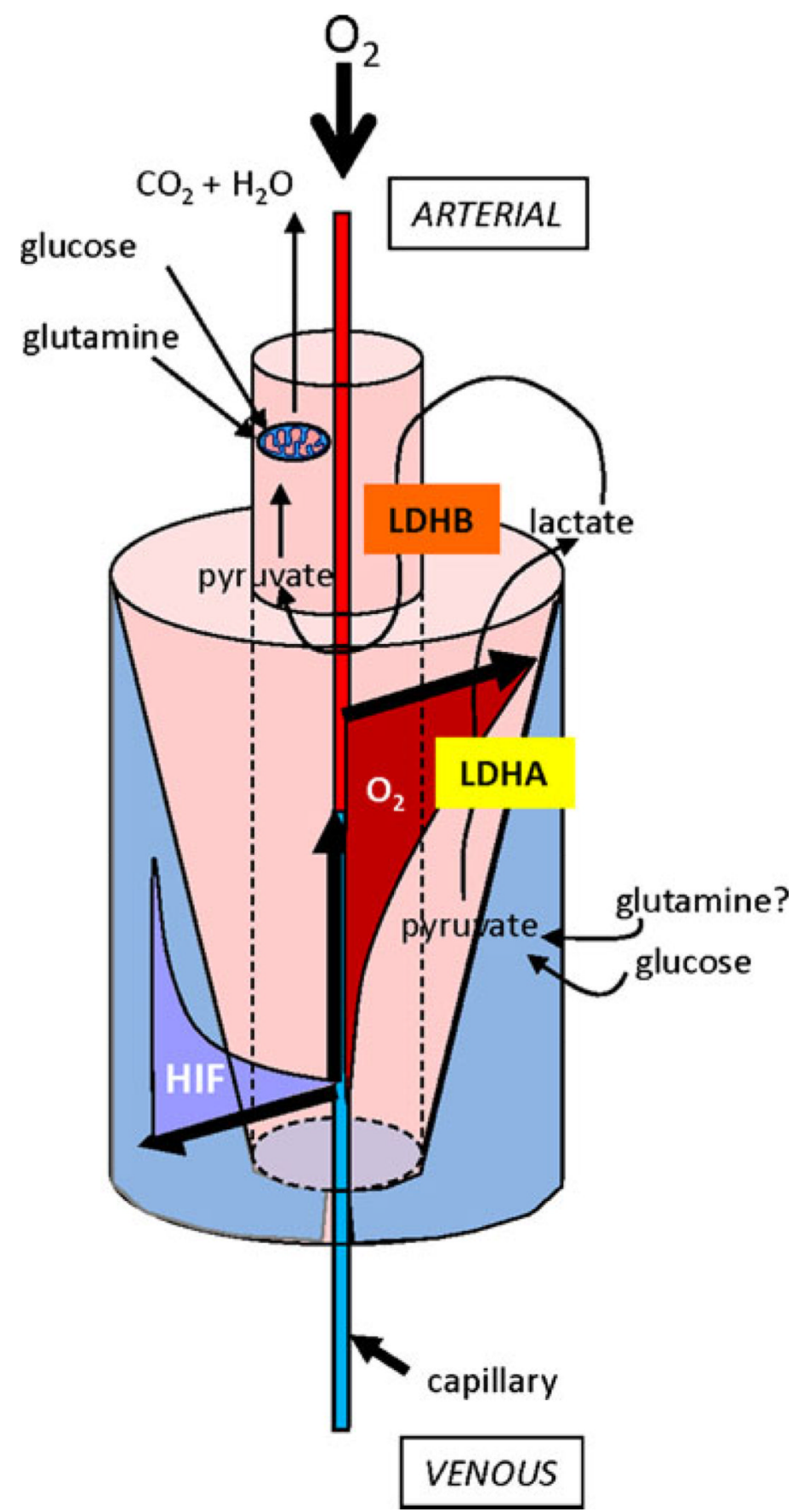

Figure 2.

Hypothetical cartoon of the tumor tissue perfused by a tumor blood vessel. A threedimensional cutout of a tumor tissue block with a central capillary feeding an inner layer of cells from the arterial side with oxygen and nutrients. The layer of cells proximal to the arterial side consume oxygen with glucose and glutamine serving as substrates, causing the venous blood to be deprived of oxygen and rendering the cancer cells toward the venous side hypoxic. Hypoxia, as illustrated by the oxygen gradient decreasing away from the arterial side of the central capillary, induces HIF-1 if it not already stabilized by upstream oncogenic pathways. Deregulated HIF-1 along with oncogenes, such as Myc, can induce the Warburg effect. Note that lactate produced by LDHA in the hypoxic cuff of cells is 
converted to pyruvate by LDHB in the central layer of cells for oxidation in the mitochondrion 